\title{
Numerical Investigation of a Kretschmann-Type Surface Plasmon Resonance Waveguide Sensor
}

\author{
Jun Shibayama, Member, IEEE, Member, OSA, Taichi Takeuchi, Naoki Goto, \\ Junji Yamauchi, Member, IEEE, Member, OSA, and Hisamatsu Nakano, Fellow, IEEE
}

\begin{abstract}
A Kretschmann-type surface plasmon resonance waveguide sensor is analyzed using the wide-angle beampropagation method (BPM) with the complex Padé approximant and the finite-difference time-domain (FDTD) methods based on the recursive convolution (RC) and piecewise linear RC (PLRC) techniques. The wavelength responses of the sensor are calculated, and a detailed comparison of the numerical results is made. The BPM results are validated through a comparison with the FDTD results, in which the PLRC technique is required in terms of accuracy. The waveguide sensor shows a maximum absorption wavelength shift from 0.609 to $0.623 \mu \mathrm{m}$, as the refractive index of an analyte is increased from 1.330 to 1.334 , which is comparable to the sensitivity of the conventional Kretschmann configuration.
\end{abstract}

Index Terms-Beam-propagation method (BPM), finitedifference time-domain (FDTD) method, optical waveguide, Padé approximant, piecewise linear recursive convolution (PLRC), surface plasmon resonance (SPR) sensor.

\section{INTRODUCTION}

A SURFACE plasmon resonance (SPR) optical sensor based on the Kretschmann configuration has been studied in various applications, such as chemical and biochemical sensing, drug screening, and environmental monitoring [1]-[4]. Although the Kretschmann-based sensor allows real-time and high-sensitivity measurements, its miniaturization is difficult due to the bulk structure that is composed of a prism coated with a thin metal. To miniaturize an SPR sensor, a planar waveguide structure has received much attention [1], [5], [6]. Unfortunately, the sensitivity of the waveguide sensor is generally not as high as that of the Kretschmann-based sensor.

To circumvent these difficulties, we have briefly reported a sensor structure, in which input/output parts in the Kretschmann configuration are replaced with optical waveguides [7]. The characteristics of the Kretschmann-type waveguide-based SPR sensor have been analyzed in the frequency domain using the wide-angle beam-propagation method (BPM) [8], [9] with the complex Padé approximant [10]-[12]. However, the numerical results of the BPM have not thoroughly been validated using more general time-domain approaches, such as the finite-difference time-domain (FDTD) method.

Manuscript received January 11, 2007; revised June 24, 2007. This work was supported in part by the Takahashi Industrial and Economic Research Foundation.

J. Shibayama, N. Goto, J. Yamauchi, and H. Nakano are with the Faculty of Engineering, Hosei University, Tokyo 184-8584, Japan (e-mail: shiba@ hosei.ac.jp; j.yma@hosei.ac.jp; nakano@hosei.ac.jp).

T. Takeuchi is with Sony Corporation, Tokyo 108-0075, Japan.

Color versions of one or more of the figures in this paper are available online at http://ieeexplore.ieee.org.

Digital Object Identifier 10.1109/JLT.2007.903823
In the time-domain analysis, the frequency-dependent formulation is mandatory since the metal is a dispersive material. The recursive convolution (RC) technique [13] is one of the frequently used methods. The piecewise linear RC (PLRC) technique [14], which is the improved version of the RC technique, is also widely used. The auxiliary differential equation technique [15], [16] is a simple way to take into account the material dispersion, although it requires more memory than the RC and PLRC techniques.

So far, only a few efforts have been directed at applying the frequency-dependent FDTD [(FD $\left.)^{2} \mathrm{TD}\right]$ to the analysis of SPR sensors, e.g., [17]. One reason for a lack of $(\mathrm{FD})^{2} \mathrm{TD}$ applications stems from the fact that the conventional Kretschmannbased sensors can be efficiently designed with the three-layer Fresnel equation approach based on the solution to plane wave incidence. Another reason is that the wavelength responses are not necessary for most conventional sensors since they are based on an angular interrogation scheme, where the angle of incidence of light is varied at a fixed operation wavelength. In contrast, for the waveguide sensor, an angular spread of the incident beam from the input waveguide should be fully taken into account, to which the Fresnel equation approach is not generally applicable. In addition, the wavelength response is required due to a wavelength interrogation scheme, where the operation wavelength is varied at a fixed incident angle. To address these issues, we are motivated to apply the versatile (FD) ${ }^{2}$ TDs to the analysis of the wavelength responses of the waveguide-based SPR sensors. Although the (FD) ${ }^{2}$ TDs are computationally intensive, the application is expected to provide useful data not only for the validation of the BPM results but also for the practical design of the SPR sensors.

The purpose of this paper is to discuss in detail the numerical results of the waveguide-based SPR sensor for the frequencyand time-domain analyses. In particular, the (FD) ${ }^{2}$ TDs based on the RC and PLRC techniques are applied to the analysis of the sensor, and their numerical results are compared with those of the BPM. To make the discussion self-contained, we first present the numerical methods to be used, in which we describe how to alleviate numerical instabilities that often occur when the BPM is used to analyze waveguides with the metal. We next calculate the wavelength responses of the SPR sensor, in which the convergence of the numerical results of each method is investigated with the help of an extrapolation technique. It is shown that the response obtained from the PLRC-FDTD agrees well with that from the BPM. In contrast, the response from the RC-FDTD deviates from the others. As a result, the BPM results are validated, and the PLRC-FDTD is required 


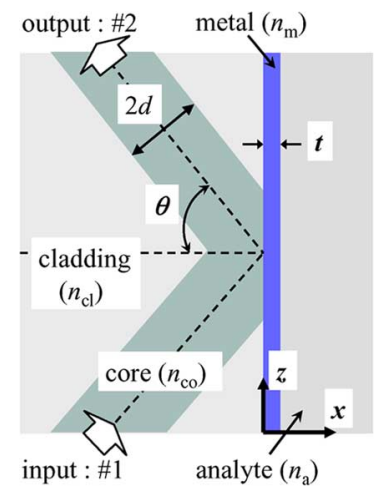

Fig. 1. Configuration of a waveguide-based SPR sensor.

for the SPR sensor analysis in terms of accuracy. We further evaluate the characteristics of the SPR sensor, showing the sensitivity comparable to that of the conventional Kretschmannbased sensor. Finally, we present the wavelength response for the experimentally obtained refractive index of the metal in the Appendix.

\section{SEnsor STRUCTURE}

Fig. 1 illustrates the configuration of the waveguide-based SPR sensor to be studied. We excite the field of the fundamental transverse magnetic (TM) mode from waveguide 1 and extract the output power in waveguide 2. Varying the operation wavelength $\lambda$, we evaluate the response of the output power, which depends on the refractive index of an analyte $n_{a}$. Water is selected as an analyte. The refractive indexes of the core and cladding are chosen to be 1.4675 and 1.46 , respectively. The refractive index of the metal $(\mathrm{Au})$ is determined using the following Drude model dielectric function:

$$
n_{m}^{2}=1+\frac{\omega_{p}^{2}}{\omega\left(j \nu_{c}-\omega\right)}
$$

with $n_{m}=0.131-j 3.654$ at $0.6328 \mu \mathrm{m}$ [4], in which $\omega$ is the angular frequency, $\omega_{p}$ is the electron plasma frequency, and $\nu_{c}$ is the effective electron collision frequency (the wavelength response using the measured refractive indexes will be compared with that based on the Drude model in the Appendix). In this case, we obtain $\omega_{p}=1.130 \times 10^{16} \mathrm{rad} / \mathrm{s}$ and $\nu_{c}=$ $1.988 \times 10^{14} \mathrm{rad} / \mathrm{s}$. Although the refractive indexes may show temperature dependence, we ignore throughout this paper the refractive index change that is caused by the thermal effect for simplicity. The core width is $2 d=1.94 \mu \mathrm{m}$, where the normalized frequency is $V \simeq 1.5$ at $\lambda=0.6 \mu \mathrm{m}$. The thickness of the metal is $t=0.045 \mu \mathrm{m}$ for the efficient coupling between the waveguide and surface plasmon polariton (SPP) modes. The analyte is sufficiently thick $(1 \mu \mathrm{m})$ to yield a converged solution. The angle of incidence of the dielectric waveguide to the metal film is designated as $\theta$.

In the configuration of Fig. 1, when the $z$-component of the effective index of the TM mode in the dielectric waveguide is equivalent to the real part of that of the SPP wave, i.e., $n_{\mathrm{TM}} \sin \theta=n_{\mathrm{SPP}}$, the resonance condition is achieved, and the incident light is coupled to the SPP wave. This results in a noticeable reduction in the output power. In [7], we have calculated $n_{\mathrm{SPP}}$ against $n_{a}$ through the eigenmode analysis using the imaginary-distance BPM [18] to find the resonance condition. As a result, we have chosen the incidence angle to be $\theta=78^{\circ}$, in which the sensor is intended to operate around $0.6 \mu \mathrm{m}[7]$.

\section{Numerical Method}

\section{A. Wide-Angle BPM With Complex Padé Approximant}

The BPM can be used to treat the reflection at the interface between different materials, provided that the interface is placed parallel to the propagating beam direction, and the angle of incidence to the interface is relatively large (this technique has been first adopted in the analysis of the reflection at a dielectric-air interface [19]). In this section, we place the SPR sensor structure, as shown in Fig. 1, and investigate the sensing characteristics using the wide-angle BPM based on the Padé approximant [8], [9].

The basic equation of the $(1,1)$ Padé-based BPM for the TM mode is expressed as

$$
\frac{\partial H_{y}}{\partial z}=\frac{\frac{1}{2 j k n_{0}}\left(D_{x x}+\nu\right)}{1+\frac{1}{4 k^{2} n_{0}^{2}}\left(D_{x x}+\nu\right)} H_{y}
$$

where

$$
\begin{aligned}
D_{x x} H_{y} & =\frac{\partial}{\partial x}\left(\frac{1}{n^{2}} \frac{\partial H_{y}}{\partial x}\right) \\
\nu & =k^{2}\left(n^{2}-n_{0}^{2}\right)
\end{aligned}
$$

in which $k$ is the free-space wavenumber, $n$ is the refractive index, and $n_{0}$ is the reference refractive index to be appropriately chosen. After discretizing (2) with the Crank-Nicolson scheme and applying the modified second-order finite-difference (FD) formula [20], we obtain a tridiagonal matrix that can be efficiently solved by the Thomas algorithm.

Here, we refer to a stability problem with respect to the sampling width in the propagation direction $\Delta z$ for the conventional wide-angle BPM. With $\Delta z$ being larger than $\simeq 0.3 \mu \mathrm{m}$, stable results can be obtained for the analysis of the present sensor. Unfortunately, we have often encountered numerical instabilities in the vicinity of the metal with a small $\Delta z$ that is comparable to $\Delta x$ to be used. These small sampling widths will be required to fairly compare the numerical accuracy of the BPM with that of the FDTDs in the next section. To suppress the instabilities that are caused by the use of a small $\Delta z$, we resort to the complex Padé approximant [10]-[12]. The complex reference refractive index approach [12], which is equivalent to the branch-cut rotation method [11], is adopted, in which the reference refractive index is changed into a complex value, i.e., $n_{0}=\bar{n}_{0} e^{i \rho}$, where $\bar{n}_{0}$ is the original real reference refractive index, and $\rho$ is a phase factor.

Preliminary calculations have shown that the complex reference refractive index approach works at wavelengths longer than the maximum absorption wavelength. However, the approach gives rise to a strong damping effect at shorter wavelengths, resulting in an underestimation of the output power. 
This damping effect is found to be caused by the attenuation factor in the term regarding the phase variation $\nu$ in (2). Hence, to avoid this effect, we empirically apply the complex reference refractive index to only $n_{0}$ in $2 j k n_{0}$ and $4 k^{2} n_{0}^{2}$ in (2), while $n_{0}$ in $\nu$ is kept real. To show the effectiveness of this modified approach, we have analyzed a four-layer planar sensor structure (cladding/core/metal/analyte). The material of each layer is the same as that used in Fig. 1, and the thicknesses of the core and metal are 1.94 and $0.045 \mu \mathrm{m}$, respectively. For the modified approach, the propagation loss is calculated to be $10.1 \mathrm{~dB} / \mathrm{mm}$ for $n_{a}=1.330$ at $\lambda=0.5 \mu \mathrm{m}$, while for the eigenmode analysis with the imaginary-distance BPM [18], the loss is $11.1 \mathrm{~dB} / \mathrm{mm}$. As a result, this modified approach can practically be used to investigate the sensor characteristics even at shorter wavelengths without the numerical instability, although its theoretical validation is not clear at present.

\section{B. $(F D)^{2} T D$ Methods}

Although the (FD) ${ }^{2}$ TDs have widely been used to analyze plasmonic structures, their applications to the analysis of the SPR sensors have been limited to only a few cases of the conventional Kretschmann structures. In particular, the wavelength responses of the waveguide-based SPR sensors have not been investigated using the FDTD, in spite of the fact that the wavelength response can be obtained by one-time solution using the pulse excitation scheme. Note that the FDTD analysis can be performed even in the case of a small incident angle $\theta$ (see Fig. 1), to which the BPM cannot be applied. These facts encourage us to examine the application of the $(\mathrm{FD})^{2} \mathrm{TDs}$, although they require computational efforts relative to the BPM.

In this paper, we perform the analysis using the (FD) ${ }^{2} \mathrm{TDs}$ based on the RC and PLRC techniques for the Drude model dielectric function (1). It is assumed that, for the RC technique, the electric field is constant over $\Delta t$, while, for the PLRC technique, the electric field has piecewise linear functional dependence over $\Delta t$. Therefore, the PLRC technique may achieve better accuracy than the RC technique.

For the PLRC-FDTD, the update equation for the $\boldsymbol{E}$ field in the metal is expressed as

$$
\begin{aligned}
\boldsymbol{E}^{n+1}= & \frac{\epsilon_{\infty}-\xi^{0}}{\epsilon_{\infty}+\chi^{0}-\xi^{0}} \boldsymbol{E}^{n}+\frac{1}{\epsilon_{\infty}+\chi^{0}-\xi^{0}} \phi^{n} \\
& +\frac{\Delta t / \epsilon_{0}}{\epsilon_{\infty}+\chi^{0}-\xi^{0}} \nabla \times \boldsymbol{H}^{n+1 / 2}
\end{aligned}
$$

where

$$
\begin{aligned}
\phi^{n} & =\left(\Delta \chi^{0}-\Delta \xi^{0}\right) \boldsymbol{E}^{n}+\Delta \xi^{0} \boldsymbol{E}^{n-1}+e^{-\nu_{c} \Delta t} \phi^{n-1} \\
\chi^{0} & =\frac{\omega_{p}^{2}}{\nu_{c}}\left[\Delta t-\frac{1}{\nu_{c}}\left(1-e^{-\nu_{c} \Delta t}\right)\right] \\
\Delta \chi^{0} & =-\frac{\omega_{p}^{2}}{\nu_{c}^{2}}\left(1-e^{-\nu_{c} \Delta t}\right)^{2} \\
\xi^{0} & =\frac{\omega_{p}^{2}}{\nu_{c}}\left[\frac{\Delta t}{2}-\frac{1}{\nu_{c}^{2} \Delta t}\left(1-e^{-\nu_{c} \Delta t}\right)+\frac{1}{\nu_{c}} e^{-\nu_{c} \Delta t}\right] \\
\Delta \xi^{0} & =-\frac{\omega_{p}^{2}}{\nu_{c}^{2}}\left[\frac{1}{\nu_{c} \Delta t}\left(1-e^{-\nu_{c} \Delta t}\right)^{2}-\left(1-e^{-\nu_{c} \Delta t}\right) e^{-\nu_{c} \Delta t}\right]
\end{aligned}
$$

with $\epsilon_{\infty}=1$ for a Drude model. To obtain the $\boldsymbol{H}$ field, the standard update equations are used. Note that (3) reduces to the $\mathrm{RC}$ technique for $\xi^{0}=0$ and $\Delta \xi^{0}=0$. In this paper, we do not use the envelope formulation, so that we treat the total field with an optical carrier.

In the application of the $(\mathrm{FD})^{2} \mathrm{TDs}$, we should pay attention to the choice of $\omega_{p}$ and $\nu_{c}$ at the metal-dielectric interface. In the following simulation, we place the interface on the electric field component and derive $\omega_{p}$ and $\nu_{c}$ at the interface from the averaged relative permittivity between the metal and dielectric materials.

\section{ANALysis of A WaVEguide-BASEd SPR SENSOR}

We now study the wavelength response of the sensor, varying the wavelength from 0.50 to $0.70 \mu \mathrm{m}$. The refractive index of the metal is determined from (1) at a given wavelength. In the analysis using the complex-Padé-based BPM discussed in Section III, $\bar{n}_{0}$ is chosen to be $n_{\mathrm{TM}} \cos 78^{\circ}$, and $\rho$ is taken to be $-2^{\circ}$ for the metal region and 0 for the waveguide and analyte regions. For the FDTD, taking advantage of the timedomain analysis, we use the pulse excitation scheme to obtain the wavelength response, in which the center wavelength of the input pulse is $0.600 \mu \mathrm{m}$, and its bandwidth spans $0.34 \mu \mathrm{m}$ in the $1 / e$ full width that is wide enough to calculate the relatively narrow wavelength range in Fig. 2.

Fig. 2 shows the wavelength response of the normalized output power from waveguide 2 for $n_{a}=1.330$, in which Fig. 2(a)-(c) represent the results for the BPM, RC-FDTD, and PLRC-FDTD, respectively. To investigate the convergence of the numerical results, we calculate the responses for the several spatial sampling widths. In Fig. 2(a), the BPM yields almost the same wavelength responses for the two cases. In contrast, the responses obtained from the RC-FDTD and PLRC-FDTD gradually shift rightward with a reduction in sampling widths. It is also seen that the wavelength responses from the RC-FDTD are not sharper than those from the BPM and the PLRC-FDTD.

Here, we pay attention to the maximum absorption wavelength. Fig. 3 depicts the absorption wavelength for each method as a function of $\Delta x$. It is seen that, as $\Delta x$ is reduced, the absorption wavelengths approach specific values. Note that, for the FDTDs, the results are shown for $\Delta x \geq 0.0075 \mu \mathrm{m}$ due to the limit of the computational memory. Therefore, the convergence with respect to the sampling width has not been obtained for the FDTD results. To further investigate the effect of the sampling widths on the wavelength response, we next discuss an extrapolated value of the maximum absorption wavelength. To obtain the extrapolated value, we derive the second-order function using the two successive values of the absorption wavelengths, i.e., the values with $\Delta x=0.005$ and $0.0075 \mu \mathrm{m}$ are used for the BPM, and those with $\Delta x=0.0075$ and $0.009 \mu \mathrm{m}$ are used for the FDTDs. In addition, the extrapolated value at $\Delta x=0$ for each method is shown as a straight line. It is found that the extrapolated value of the PLRC-FDTD $(0.6088 \mu \mathrm{m})$ agrees well with that of the BPM $(0.6094 \mu \mathrm{m})$, while the value of RC-FDTD shows a slight overestimation $(0.6108 \mu \mathrm{m})$. 


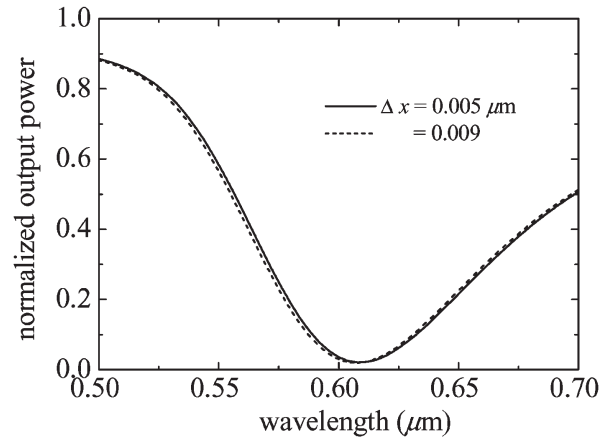

(a)

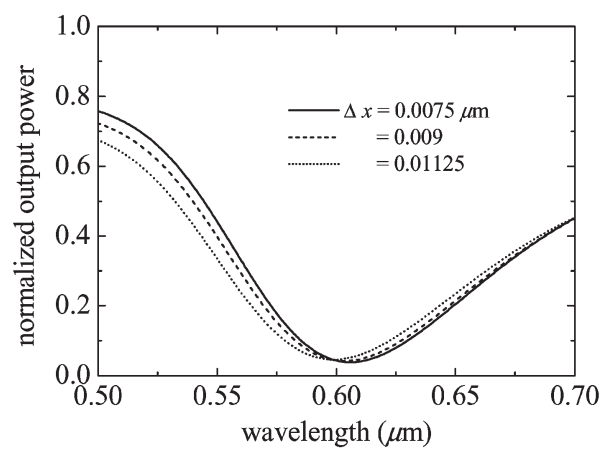

(b)

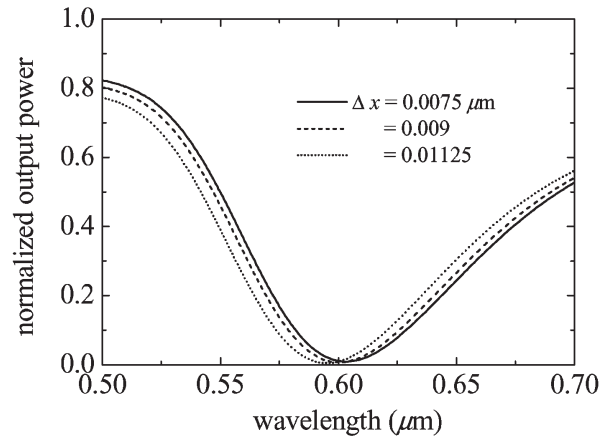

(c)

Fig. 2. Wavelength response for (a) BPM, (b) RC-FDTD, and (c) PLRC-FDTD. $\Delta z=\Delta x \tan \theta / 2.5$.

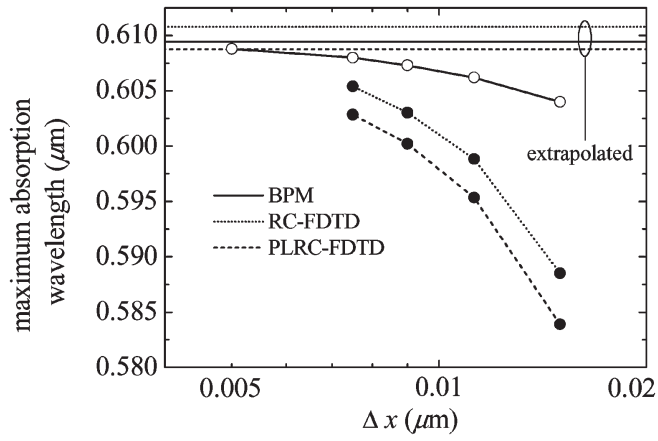

Fig. 3. Maximum absorption wavelength as a function of sampling width. $\Delta z=\Delta x \tan \theta / 2.5$. For the FDTDs, we only show the results for $\Delta x \geq$ $0.0075 \mu \mathrm{m}$, due to the limit of the computational memory.

Further calculations using the results in Fig. 3 readily show that each method is spatially second-order accurate, even in the presence of the metal. Note, however, that the error of the FDTD is larger than that of the BPM by an order of magnitude,

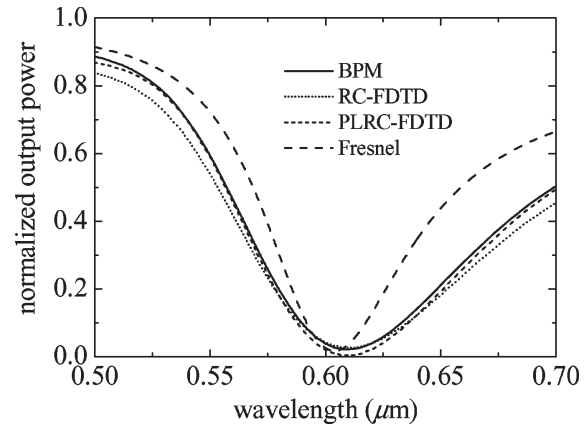

Fig. 4. Extrapolated wavelength response. The result obtained from the Fresnel equation approach is also included.

as expected in Fig. 3. This is due to the fact that the FD equation for the material interface in Yee's mesh coincides with Stern's formula [21], [22], whose accuracy is slightly less than that of the modified second-order FD formula used in the BPM [20]. As a result, the difference in the error is attributed to the accuracy of the FD equation to be used.

The convergence of the numerical result is studied not only for the absorption wavelength discussed previously but also for the other wavelengths. The overall spectral range of the response shown in Fig. 2 is extrapolated in Fig. 4. It can be seen that the response of the PLRC-FDTD is in close agreement with that of the BPM. In contrast, the RC-based result deviates from the others. Consequently, the BPM results are validated in comparison with the FDTD results. Besides, the RC technique induces an appreciable error, and the PLRC technique is necessary in terms of the accuracy of the numerical results.

Note that the conventional Kretschmann-based SPR sensor has been modeled using the three-layer Fresnel equation approach [23], [17]. In Fig. 4, the result of the Fresnel approach is also included, in which the waveguide region $(x<0)$ is assumed to be a homogeneous medium [7]. The maximum absorption wavelength is calculated to be $0.6038 \mu \mathrm{m}$, which is in reasonable agreement with the wavelengths obtained from the BPM and the FDTDs. However, the Fresnel approach cannot fully predict the wavelength response of the present SPR sensor, as shown in Fig. 4, since the approach is based on plane wave incidence, which cannot treat an angular spread of the incident beam of the present SPR sensor structure. Although not illustrated, widening the core width of the present sensor results in a sharper wavelength response since the angular spread of the incident beam becomes narrower.

Fig. 5 shows the maximum absorption wavelength of the present SPR sensor as a function of $n_{a}$, in which the BPM is adopted due to its computational efficiency. Note that the extrapolated values are shown in Fig. 5. It is clear that the maximum absorption wavelength depends on $n_{a}$, in which the wavelength shifts from 0.609 to $0.623 \mu \mathrm{m}$, when the refractive index of the analyte $n_{a}$ increases by 0.004 units. This large shift is almost identical to that of the conventional sensor based on the Kretschmann configuration [2] and is detectable by an optical spectrum analyzer.

The field distribution at a maximum absorption wavelength of $0.609 \mu \mathrm{m}$ for $n_{a}=1.330$ is shown in Fig. 6(a), which is calculated using the BPM. It is observed that the SPP wave 


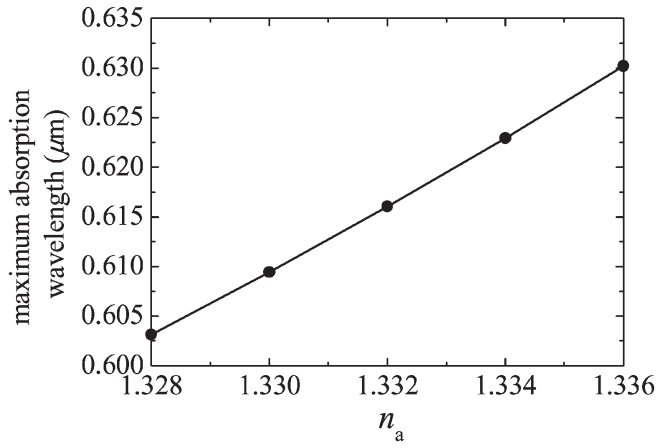

Fig. 5. Maximum absorption wavelength as a function of analyte index $n_{a}$.

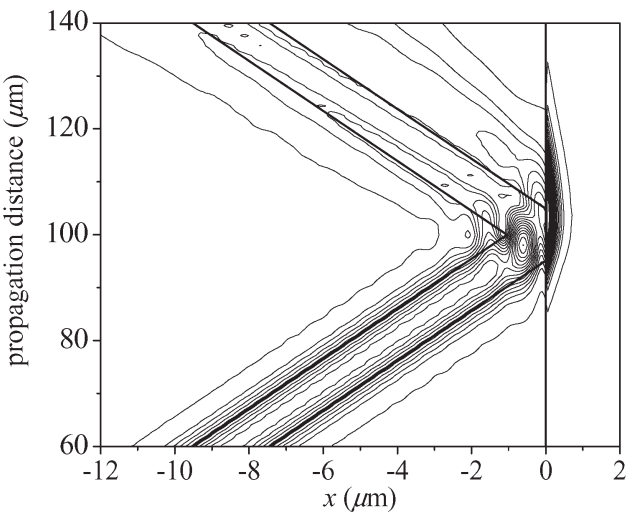

(a)

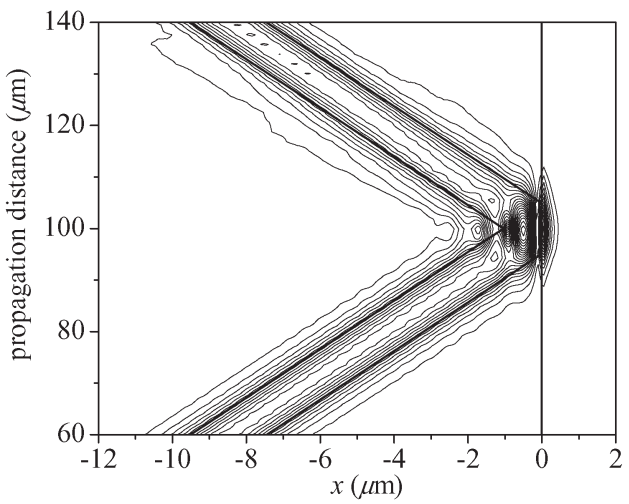

(b)

Fig. 6. Field distributions for $n_{a}=1.330$ obtained from the BPM. (a) $\lambda=$ $0.609 \mu \mathrm{m}$ and (b) $\lambda=0.5 \mu \mathrm{m}$.

is excited along the metal-analyte interface, and its amplitude is rapidly attenuated with the leaky wave generated in the region for $x<0$. This results in a minimum reflectivity of $0.02 \%$. In contrast, when $\lambda$ is shifted from the maximum absorption wavelength, e.g., at $\lambda=0.5 \mu \mathrm{m}$, most of the power is reflected (88\%), as shown in Fig. 6(b).

From Fig. 6(a), it can be seen that the SPP wave starts to appear around $z=85 \mu \mathrm{m}$ and almost disappears around $130 \mu \mathrm{m}$. This short sensing length contributes to a reduced amount of the analyte, when compared with the conventional waveguide-based sensors, in which the sensing section is typically $2 \mathrm{~mm}$ in length [5], [6]. In addition, the present sensor shows a narrow spatial profile of the incident beam, i.e., the full-width at half-maximum of the beam is calculated to be 3.8 wavelengths at $0.633 \mu \mathrm{m}$, while for the Kretschmann configuration, it is said to be 7.4 wavelengths [17]. Taking advantage of this narrow profile of the waveguide-based sensor, we expect to reduce the interaction between the sensors when they are vertically stacked for multichannel sensing. The application to 3-D models of multichannel waveguide-based sensors is an interesting subject and is left for future study.

\section{CONCLUSION}

We have numerically investigated a Kretschmann-type SPR waveguide sensor in the frequency and time domains. The RC and PLRC techniques have been applied to the time-domain analysis of the SPR sensor, and their results have been compared with those of the wide-angle BPM with the complex Padé approximant. After explaining the numerical methods that were used, we calculate the wavelength responses of the waveguide sensor and study the convergence of the numerical results. A close correspondence is found between the BPM and PLRC-FDTD results, showing the validity of the BPM results and the necessity of the PLRC technique in terms of accuracy. It is also shown that the Fresnel equation approach, which is frequently used for modeling the conventional Kretschmannbased sensor, cannot fully predict the wavelength response of the present waveguide sensor. The waveguide sensor exhibits the sensitivity comparable to that of the conventional Kretschmann-based sensor and therefore could be a potential candidate for an SPR sensor that is integrated into optical circuits. A Kretschmann-type SPR waveguide sensor with a sharper dip in wavelength response is now under study.

\section{APPENDIX \\ COMPARISON OF THE WAVELENGTH RESPONSE USING Measured Refractive Indexes of the Metal AND THAT USING THE DRUdE MODEL}

In this paper, we have investigated the wavelength responses of the waveguide-based SPR sensor with the help of the Drude model for the material dispersion of the metal. Here, we compare the response using the measured refractive indexes of the metal available in [24] and that using the Drude model that was determined with one of the measured refractive indexes. The complex Padé-based BPM is used to analyze the same sensor structure as that in Fig. 1, except for the refractive index of the metal.

Fig. 7 shows the wavelength response for $n_{a}=1.330$. The black circles indicate the responses that were obtained using the measured refractive indexes, in which the data for the imaginary parts of the refractive indexes are missed around $0.6 \mu \mathrm{m}$ in [24]. To show the overall wavelength response, we use the interpolated refractive indexes that were obtained from available values. The overall response is indicated by the broken line. The black line represents the response based on the Drude model, in which one of the measured indexes, i.e., $n_{m}=0.166-j 3.15$ at $0.6526 \mu \mathrm{m}$, is used for (1). Note that the maximum absorption wavelengths in Fig. 7 shift toward longer wavelengths, compared with the wavelength discussed in Section IV. This is caused by the difference of the refractive 


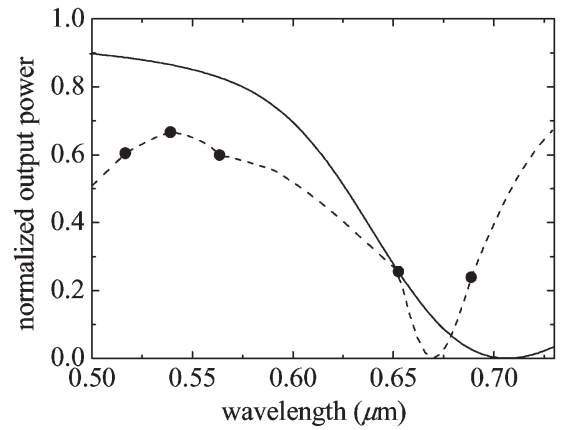

Fig. 7. Wavelength response.

indexes that were used for each case, which affects the resonance condition of the SPP wave.

It is seen that the maximum absorption wavelength using the Drude model (solid line) is in reasonable agreement with that using the interpolated value (broken line). Nevertheless, the response of the former does not perfectly follow that of the latter one. This is due to the fact that the Drude model cannot fully describe the measured refractive indexes, particularly at the shorter wavelengths [25]. To obtain a more accurate description of the dispersion of the metal, the extended Drude model with an additional Lorentzian term may be used [25], whose applications to waveguide-based SPR sensors in both frequency and time domains will be addressed in future work.

\section{ACKNOWLEDGMENT}

The authors would like to thank Dr. T. Kashiwa of Kitami Institute of Technology for invaluable discussions regarding the $(\mathrm{FD})^{2} \mathrm{TD}$ methods and T. Yamazaki of Hosei University for his technical discussions.

\section{REFERENCES}

[1] J. Homola, S. S. Yee, and G. Gauglitz, "Surface plasmon resonance sensors: Review," Sens. Actuators B, Chem., vol. 54, no. 1, pp. 3-15, Jan. 1999.

[2] L. M. Zhang and D. Uttamchandani, "Optical chemical sensing employing surface-plasmon resonance," Electron. Lett., vol. 24, no. 23, pp. 14691470, Nov. 1988.

[3] K. Matsubara, S. Kawata, and S. Minami, "Optical chemical sensor based on surface-plasmon measurement," Appl. Opt., vol. 27, no. 6, pp. 1160-1163, Mar. 1988.

[4] Ş. K. Özdemir and G. Turhan-Sayan, "Temperature effects on surface plasmon resonance: Design considerations for an optical temperature sensor," J. Lightw. Technol, vol. 21, no. 3, pp. 805-814, Mar. 2003.

[5] R. D. Harris and J. S. Wilkinson, "Waveguide surface plasmon resonance sensors," Sens. Actuators B, Chem., vol. 29, no. 1-3, pp. 261-267, Oct. 1995.

[6] J. Čtyroký, J. Homola, and M. Skalsky, "Tuning of spectral operation range of a waveguide surface plasmon resonance sensor," Electron. Lett, vol. 33, no. 14, pp. 1246-1248, Jul. 1997.

[7] J. Shibayama, T. Takeuchi, T. Yamazaki, J. Yamauchi, and H. Nakano, "Analysis of a surface plasmon resonance waveguide sensor using the BPM with the complex Padé approximant," in Proc. Integr. Photon. Res. Appl. Conf., IME4, Uncasville, CT, Apr. 2006.

[8] D. Yevick and M. Glasner, "Analysis of forward wide-angle light propagation in semiconductor rib waveguides and integrated-optic structures," Electron. Lett., vol. 25, no. 23, pp. 1611-1613, Nov. 1989.

[9] G. R. Hadley, "Wide-angle beam propagation using Padé approximant operators," Opt. Lett., vol. 17, no. 20, pp. 1426-1428, Oct. 1992.

[10] Y. Y. Lu, "A complex coefficient rational approximation of root $1+\mathrm{x}$," Appl. Numer. Math., vol. 27, no. 2, pp. 141-154, Jun. 1998
[11] H. El-Refaei, I. Betty, and D. Yevick, "The application of complex Padé approximants to reflection at optical waveguide facets," IEEE Photon. Technol. Lett., vol. 12, no. 2, pp. 158-160, Feb. 2000.

[12] H. L. Rao, M. J. Steel, R. Scarmozzino, and R. M. Osgood, Jr., "Complex propagators for evanescent waves in bidirectional beam propagation method," J. Lightw. Technol., vol. 18, no. 8, pp. 1155-1160, Aug. 2000.

[13] R. Luebbers, F. P. Hunsberger, K. S. Kunz, R. B. Standler, and M. Schneider, "A frequency-dependent finite-difference time-domain formulation for dispersive materials," IEEE Trans. Electromagn. Compat., vol. 32, no. 3, pp. 222-227, Aug. 1990.

[14] D. F. Kelley and R. J. Luebbers, "Piecewise linear recursive convolution for dispersive media using FDTD," IEEE Trans. Antennas Propag., vol. 44, no. 6, pp. 792-797, Jun. 1996.

[15] T. Kashiwa and I. Fukai, "A treatment by the FD-TD method of the dispersive characteristics associated with electronic polarization," Microw. Opt. Technol. Lett., vol. 3, no. 6, pp. 203-205, Jun. 1990.

[16] R. M. Joseph, S. C. Hagness, and A. Taflove, "Direct time integration of Maxwell equations in linear dispersive media with absorption for scattering and propagation of femtosecond electromagnetic pulses," Opt. Lett., vol. 16 , no. 18 , pp. 1412-1414, Sep. 1991.

[17] D. Christensen and D. Fowers, "Modeling SPR sensors with the finitedifference time-domain method," Biosens. Bioelectron., vol. 11, no. 6/7, pp. 677-684, 1996.

[18] J. Shibayama, T. Yamazaki, J. Yamauchi, and H. Nakano, "Eigenmode analysis of a light-guiding metal line loaded on a dielectric substrate using the imaginary-distance beam-propagation method," J. Lightw. Technol., vol. 23, no. 3, pp. 1533-1539, Mar. 2005.

[19] Y. Chung and N. Dagli, "Analysis of integrated optical corner reflectors using a finite-difference beam propagation method," IEEE Photon. Technol. Lett., vol. 3, no. 2, pp. 150-152, Feb. 1991.

[20] J. Yamauchi, G. Takahashi, and H. Nakano, "Modified finite-difference formula for semivectorial $\mathrm{H}$-field solutions of optical waveguides," IEEE Photon. Technol. Lett, vol. 10, no. 8, pp. 1127-1129, Aug. 1998.

[21] M. S. Stern, "Semivectorial polarized finite difference method for optical waveguides with arbitrary index profiles," Proc. Inst. Elect. Eng., J, vol. 135, no. 1, pp. 56-63, Feb. 1988.

[22] J. Yamauchi, T. Mugita, and H. Nakano, "Implicit Yee-mesh-based finitedifference full-vectorial beam-propagation method," J. Lightw. Technol., vol. 23, no. 5, pp. 1947-1955, May 2005.

[23] W. P. Chen and J. M. Chen, "Use of surface plasma waves for determination of the thickness and optical constants of thin metallic films," J. Opt Soc. Amer., vol. 71, no. 2, pp. 189-191, Feb. 1981.

[24] D. W. Lynch and W. R. Hunter, "Comments on the optical constants of metals and an introduction to data for several metals," in Handbook of Optical Constants of Solids, E. D. Palik, Ed. Boston, MA: Academic, 1985.

[25] A. Vial, A.-S. Grimault, D. Macías, D. Barchiesi, and M. L. de la Chapelle, "Improved analytical fit of gold dispersion: Application to the modeling of extinction spectra with a finite-difference time-domain method," Phys. Rev. B, Condens. Matter, vol. 71, no. 8, pp. 085 416-1085 416-7, Feb. 2005

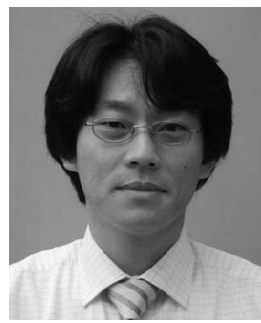

Jun Shibayama (M'03) was born in Kashiwa, Japan, on July 1, 1969. He received the B.E., M.E., and Dr.E. degrees from Hosei University, Tokyo, Japan, in 1993, 1995, and 2001, respectively.

In 1995, he joined Opto-Technology Laboratory (currently FITEL Photonics Laboratory), Furukawa Electric Co., Ltd., Ichihara, Japan. Since 1999, he has been an Assistant with the Faculty of Engineering, Hosei University. His research interests include the numerical analysis of optical waveguides.

Dr. Shibayama is a member of the Optical Society of America and the Institute of Electronics, Information, and Communication Engineers of Japan. 


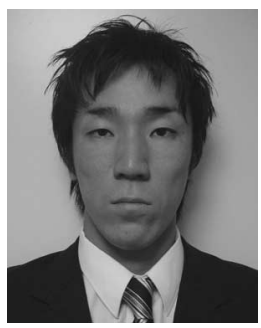

Taichi Takeuchi was born in Yokohama, Japan, on June 4, 1981. He received the B.E. and M.E. degrees from Hosei University, Tokyo, Japan, in 2005 and 2007, respectively.

In 2007, he joined Sony Corporation, Tokyo.

Mr. Takeuchi is a member of the Institute of Electronics, Information, and Communication Engineers of Japan.

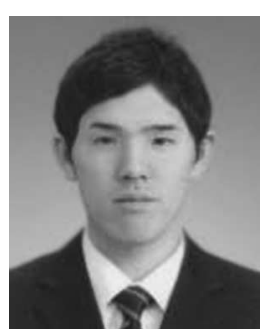

Naoki Goto was born in Tokyo, Japan, on March 4, 1984. He received the B.E. degree from Hosei University, Tokyo, Japan, in 2006. He is currently working toward the M.E. degree with the Faculty of Engineering, Hosei University.

Mr. Goto is a Student Member of the Institute of Electronics, Information, and Communication Engineers of Japan.

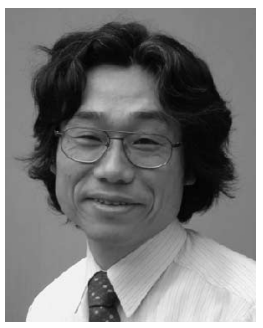

Junji Yamauchi (M'85) was born in Nagoya, Japan, on August 23, 1953. He received the B.E., M.E., and Dr.E. degrees from Hosei University, Tokyo, Japan, in 1976, 1978, and 1982, respectively.

From 1984 to 1988 , he served as a Lecturer with the Department of Electrical Engineering, Tokyo Metropolitan Technical College. Since 1988, he has been a member of the Faculty of Engineering, Hosei University, where he is currently a Professor of electronic informatics. He is the author of Propagating Beam Analysis of Optical Waveguides (Baldock, Hertfordshire, U.K.: Research Studies, 2003). His research interests include optical waveguides and circularly polarized antennas.

Dr. Yamauchi is a member of the Optical Society of America and the Institute of Electronics, Information, and Communication Engineers of Japan.

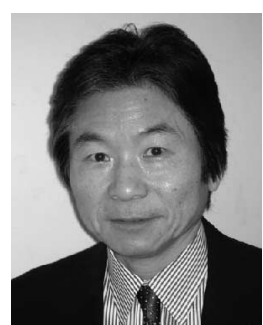

Hisamatsu Nakano (M'75-SM'87-F'92) was born in Ibaraki, Japan, on April 13, 1945. He received the B.E., M.E., and Dr.E. degrees in electrical engineering from Hosei University, Tokyo, Japan, in 1968 1970 , and 1974, respectively.

Since 1973, he has been a member of the Faculty of Engineering, Hosei University, where he is currently a Professor of electronic informatics. He has published more than 200 refereed journal papers, more than 200 international symposium papers, and more than 750 national symposium papers. He is the author of a book entitled Helical and Spiral Antennas (New York: Research Studies, Wiley, 1987), a coauthor of Analysis Methods of Electromagnetic Wave Problems, Volume Two (Norwood, MA: Artech House, 1986), and the author of Helical and Spiral Antennas, Encyclopedia of Communication (New York: Wiley, 2002). His research interests include numerical methods for low- and high-frequency antennas and optical waveguides.

Prof. Nakano is an Associate Editor of several journals and magazines, such as Electromagnetics, IEEE Antennas and Propagation Magazine, IEEE ANTENNAS AND WIRELESS PROPAG ATION LETTERS, and Asian InformationScience-Life. He was the recipient of the IEE International Conference on Antennas and Propagation Best Paper Award and the IEEE TRANSACTIONS ON ANTENNAS AND PROPAGATION Best Application Paper Award (H. A. Wheeler Award) in 1989 and 1994, respectively, the Award of Distinguished Technical Communication (from the Society for Technical Communication, USA) and the Science and Technology Progress Award (from Hangzhou, China) in 2001, and the Chen-To Tai Distinguished Educator Award (from the IEEE Antennas and Propagation Society) in 2006. In 1992, he was elected an IEEE Fellow for contributions to the design of spiral and helical antennas. 